\title{
Potential for Nile red dye-based analysis of microplastics from oceanic samples.
}

\author{
MICHELARAKI, M., JOSEPH, O., KARNIK, S., DEVALLA, S., MADANAN, K. \\ and PRABHU, R.
}

2020

C 2020 IEEE. Personal use of this material is permitted. Permission from IEEE must be obtained for all other uses, in any current or future media, including reprinting/republishing this material for advertising or promotional purposes, creating new collective works, for resale or redistribution to servers or lists, or reuse of any copyrighted component of this work in other works. 


\section{Potential for Nile red dye-based analysis of microplastics from oceanic samples}

\author{
Marina Michelaraki \\ James Hutton Institute \\ Aberdeen AB15 8QH, U.K.
}

\author{
Osheen Joseph \\ International School of \\ Photonics \\ Cochin University of Science \\ and Technology, Kochi, 682022
}

\author{
Kailasnath Madanan \\ International School of Photonics \\ Cochin University of Science and \\ Technology, \\ Kochi, 682022
}

\author{
Shruti Karnik \\ School of Engineering \\ Robert Gordon University \\ Aberdeen AB10 7GJ, U.K. \\ s.karnik@rgu.ac.uk
}

\author{
Sandhya Devalla \\ James Hutton Institute \\ Aberdeen AB15 8QH, U.K. \\ Sandhya.Devalla@hutton.ac.uk
}

\author{
Radhakrishna Prabhu \\ School of Engineering \\ Robert Gordon University \\ Aberdeen AB10 7GJ, U.K. \\ R.Prabhu@rgu.ac.uk
}

\begin{abstract}
The Global production of plastics has exceeded over 300 million metric tons. Billions of tons of plastic waste that is generated gets accumulated as plastic debris in soil, sediments, oceans and surface water with no obvious strategy to tackle them. The plastics disposed in the water and land ultimately disintegrate to microplastics, widely accepted as particles $<5 \mathrm{~mm}$ size. These microplastics are ubiquitous contaminants prevalent in the environment and pose great ecological hazard. Ensuring sustainability of coastal marine areas worldwide and reducing biodiversity loss has long been identified as a global challenge. However, dearth of scientific strategies and standardized protocols for fast and accurate detection of microplastics is a matter of concern and needs immediate attention. Therefore, robust, reliable and high through-put detection method for microplastics in oceanic environment is highly sought after. Quite a few studies have explored the potential of Nile red a solvatochromic dye in detection of microplastics. However, often Nile red alone cannot be used in quantifying microplastic due to false positives generated by staining of organic matter. In this work, we have adopted a method based on costaining of microplastics using Nile red dye and Methylene blue by fluorescence microscopy. We have observed that Nile red overestimated microplastic particles and this study serves as foundation to our future work.
\end{abstract}

Keywords-Microplastics, Nile Red, Methylene blue, costaining, fluorescence microscopy.

\section{INTRODUCTION}

Plastic litter is a globally acknowledged issue with mismanagement and poor disposal of plastics. Existence of microplastic (size $<5 \mathrm{~mm}$ )[1] in the aquatic ecosystem such as lakes, rivers, seawater, marine coastal areas, and oceans has garnered critical attention as it exerts toxic impact on marine organisms and planktons. Marine microplastics are mainly categorised as: (1) primary microplastics that are manufactured in $5 \mathrm{~mm}$ size or less, e.g.: micro beads, microfibers from clothing, nurdles[2] and (2) secondary microplastics that are generated due to disintegration of macroplastics (generated from plastic waste such as bottles, plastic bags, disposable cutlery etc.) influenced by ultraviolet irradiation, high temperatures, and mechanical shear forces[3], [4]. Microplastics are known to act as vectors for other environmental contaminants such as PAH, PCB, pharmaceuticals, thus enhancing their toxicities[5]. Due to accumulation of microplastics of varying size, composition, density, color and shape in the marine environment, detection of microplastics is very challenging. Microplastics that persist in the marine environment are ingested by different marine species (fish, shellfish, turtles, sharks and marine algae) resulting in potential hazards[6]-[8]. They are subsequently transferred to higher tropic levels via food chain and may pose serious threat to human health[9]. Assessing microplastics in the marine environment requires accurate sampling, identification, both qualitative and quantitative detection approaches. Nonetheless, high complexity in quantification of microplastics in the environment is due to lack of standardized protocols for analysis of microplastics for distinguishing and quantifying smaller size fractions smaller than $1 \mathrm{~mm}$ resulting in miscalculation of microplastic concentration. Visual inspection of microplastics with microscopic techniques have been employed resulting in underestimating or overestimating. Chemical identification techniques such as FTIR and Raman spectroscopy provide qualitative confirmation but implementing it for vast oceanic samples can be expensive, slow paced and tedious[10]. Method of detection of microplastics should be robust, reliable, cost-effective, offer high through-put and precisely enumerating microplastics in a diversified ecological template. Fluorescence staining methods 
have gained elevated significance due to simplistic and sensitive approach.

NR dye (9-diethylamino-5- benzo[a]phenoxazinone (Figure 1) have been used by various groups in tagging the microplastics and microfibers. It has also been occasionally used to determine the lipid content of animal cells and microorganisms, such as mammalian cells, bacteria, yeasts and microalgae[11], [12]. Maes et al. have developed NR staining method for detecting and quantifying microplastics with a possible drawback of staining organic species such as marine algae resulting in false positives[13]. Shim et al. have used wet peroxide method in combination with NR staining of microplastics in marine samples followed by further confirmation by FTIR to fully distinguish microplastics from biogenic matter[14]. Limitations of NR were clearly highlighted while quantifying microplastics in field samples due to the ability of NR in staining biological organic matter. Erni-Cassola et al. have employed $\mathrm{H}_{2} \mathrm{O}_{2}$ digestion followed by NR staining for detecting strongly hydrophobic microplastics<smiles>CCN(CC)c1ccc2nc3c4ccccc4c(=O)cc-3oc2c1</smiles>

(PP, PS, nylon 6 and PE)[15]. However, the protocol cannot be applied for detecting less hydrophobic samples such as PUR, PC, PVC, and PET which constituted $\sim 25 \%$ of the European plastic demand in 2015, as chitin and wood lignin also fluoresced when stained with Nile red resulting in false negatives. Recently, Stanton et al. have adopted a costaining approach by using NR in combination with 4',6- diamidino-2phenylindole (DAPI), which binds to biological samples[16]. This method accounted for the extent to which NR could overestimate microplastic particles using DAPI to identify DNA-specific organic matter stained by NR. Although majority did not, plastics of some colors autofluoresced in the blue wavelengths of light used to observe DAPI fluorescence. Recently, staining method utilizing fluorescent dyes - Safranine $\mathrm{T}$, fluorescein isophosphate, Nile red was developed based on the thermal expansion and contraction characteristics of the plastic[17]. Konde and coworkers have investigated Nile Red staining in combination with photoluminescence spectroscopy to detect the polymer type and to differentiate plastics from non-plastics[18].

NR, a lipophilic dye with phenoxazine nucleus (Figure 1) is solvatochromatic i.e. its fluorescence intensity and colour depend upon the relative hydrophobicity of the solvent. For instance, in the presence of midpolar solvents (e.g. dichloromethane) fluorescence is reddish while in less polar solvents (e.g. hexane) it is yellow-gold. On the other hand, the dye's fluorescence is quenched in aqueous environments thus acting as a hydrophobic probe.

In the present study, we have developed a Nile Red (NR) dye-based methodology for staining of microplastics and their detection by fluorescence microscopy. In our work, we are currently investigating a costaining approach by using methylene blue (MB)[19], a well-known biological stain as a second dye in combination with NR. In our ongoing research, we have applied this method for few oceanic soil samples where, MB binds with most of the organic or biological matter to get rid of false positives that usually result in overestimation of microplastics due to NR.

\section{MATERIALS AND METHOD}

Methylene blue stock solution (10\%) was prepared by dissolving $\mathrm{MB}$ in water and subsequently, working solutions of $0.1 \%$ and $0.6 \% \mathrm{MB}$ were used. NR stock solution of $100 \mathrm{mg} / \mathrm{l}$ was prepared in n-hexane. While preparing the NR stock solution in hexane, it was first dissolved in small amounts of acetone and then made up to a known volume in hexane. This is due to the lower solubility of NR in hexane. Working solution of $0.5 \mathrm{mg} / \mathrm{l}$ was prepared from the stock solution after suitable dilution. All NR and MB flasks were covered in aluminum foil.

Pretreatment of samples prior to detection and quantification of microplastics by NR tagging and fluorescence microscopy is necessary. Initially microplastics are separated from soils/sediments and collected onto a filter paper. A highly dense salt solution such as zinc bromide (our laboratory method), is used to separate the microplastics from soils/sediments which have a lower density to that of the salt solution. Following density separation, the microplastics (fibers and fragments) floating on the salt solution are filtered onto a Whatman filter paper, $55 \mathrm{~mm}$ dia. of $1.2 \mu \mathrm{m}$ particle size retention by vacuum filtration. For the examination of the samples, a Nikon SMZ1500 Stereosco.pic Zoom Microscope and Leica DM5000 B Fluorescence Microscope (FM) were used. The samples were examined in the FM at magnification of 10 and under three wavelengths: UV excitation $(360 / 340$ $\mathrm{nm})$ - blue emission $(470 / 40 \mathrm{~nm})$, blue excitation $(480 / 40$ $\mathrm{nm})$ - green emission $(530 / 27 \mathrm{~nm})$ and green excitation $(560 / 15 \mathrm{~nm})$ - red emission $(590 \mathrm{~nm})$. A series of blank experiments were initially performed to ensure there was no contamination or interference from the samples and dyes used.

\section{Staining with Nile red of Microplastics}

The microplastics of different polymer types were prepared from everyday products which were initially cut into small pieces. These small plastic pieces were cryo-milled into microplastics using the SPEX Sample Prep - 6775 Polypropylene (PP), Polyvinylchloride (PVC), polytetrafluoroethylene (PTFE), Polycarbonate (PC), polyethylene (PE) from biodegradable bags and polyethylene terephthalate (PET) from a bottle. The filter papers were further carefully washed with $500 \mu \mathrm{l}$ water $(50 \mu \mathrm{l}$ at a time) after about 30 mins of staining to remove excess dye. Fluorescence measurements of the samples were recorded using the settings. 


\section{Staining with Methylene Blue and Nile Red}

All the standards (PVC, PC, PTFE, PP, PET and PE) were stained with $0.1 \%$ and $0.6 \% \mathrm{MB}$ (sprayed $\sim 2$ times until the whole area was stained) followed by $200 \mu \mathrm{L}$ of NR and fluorescence were recorded after $30 \mathrm{~min}$.

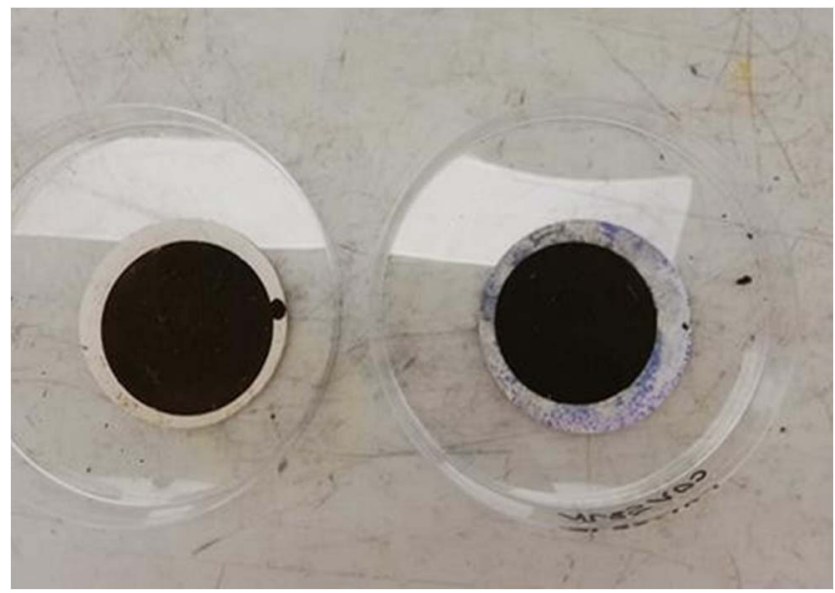

Figure 2. Oceanic sample prior (left) and upon (right) staining with both methylene blue and nile red

Considering the purpose of using MB was to minimize interference from organic matter, the experiment was conducted on two Oceanic soil samples to simulate real conditions. The samples were stained with $0.6 \% \mathrm{MB}$, followed by NR $(200 \mu \mathrm{L})$ addition (Fig. 3). The final and most important aim of this experiment is to test the utility of MB on reducing the background interference of environmental samples. Considering detection of MPs based on color is not feasible with a FM, the MPs were identified based on their shape and fluorescence either as fibers or particles/fragments. Long or curled fluorescing strips were considered as fibers and amorphous or circular fluorescing pieces were considered fragments. However, we are in process of further testing and validating this newly designed method with abroad range of oceanic samples to assess the efficacy of the process in vast environmental matrices.

\section{RESULTS AND DISCUSSIONS}

Microplastics fluoresced in all the three wavelength filters available for the fluorescence microscope-
UV excitation $(360 / 40 \mathrm{~nm})$ - blue emission $(470 / 40 \mathrm{~nm})$, blue excitation $(480 / 40 \mathrm{~nm})$ - green emission $(530 / 27 \mathrm{~nm})$ and green excitation $(560 / 15 \mathrm{~nm})$ - red emission $(590 \mathrm{~nm})$. However, Fluorescence was best observed in the green emission which is consistent with our previous work[20] and hence this wavelength emission was chosen for subsequent analysis.

Fig. 3 illustrates staining of standard PVC with NR alone and costaining with $\mathrm{MB}(0.1 \%$ and $0.6 \%)$ and NR where we try to analyze effect of concentration MB may have on the fluorescence. However, no significant difference was observed on the fluorescence produced from the two concentrations. Fig. 4 represents effect of costaining on Oceanic soil sample with $\mathrm{MB}$ and NR. Staining of oceanic samples with $0.6 \% \mathrm{MB}$ appeared to give better results than with $0.1 \% \mathrm{MB}$ We could not determine the optimal MB concentration upon addition of $\mathrm{NR}$ on the MB stained standards, but $0.6 \%$ MB clearly diminishes the fluorescence generated from biological matter more effectively. A remarkable outcome of this experiment was that the fluoresced particles standing out from the dark background which indicated that MPs fluoresce upon NR staining while MB staining reduces fluorescence of non- plastic materials.

\section{CONCLUSION}

The findings from this study represent a new methodology for detection of microplastics using coapplication of NR and MB. Our method involving a co-staining approach and FM, appears promising as MB has good biological staining ability thereby minimizing interferences and reducing false positives caused by NR alone. Detection of microplastics by staining followed by fluorescence microscopy, provides relatively cheaper and faster detection in comparison to the more expensive and time-consuming spectroscopic techniques. The method developed is also useful for providing information such as shape and size of Microplastics which would be useful for risk assessment based studies. This method might prove to be efficient in quantifying microplastics accurately. The present research provides preliminary understanding which can be further developed for the identification and quantification of microplastics in oceanic and marine environment. Our future work will be dedicated to extending this work to more rigorous assessment of various

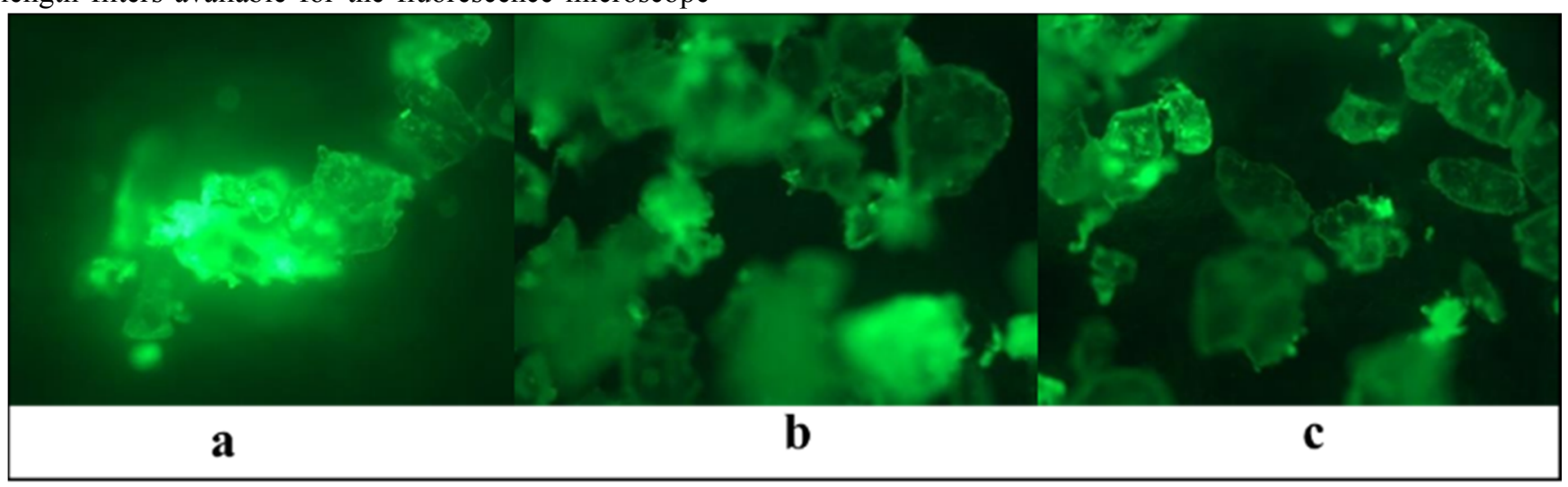

Figure 3. Staining of standard PVC with $200 \mu \mathrm{L}$ NR (a), $0.1 \% \mathrm{MB}$ and $200 \mu \mathrm{L}$ NR (b) and $0.6 \% \mathrm{MB}$ and $200 \mu \mathrm{L}$ NR (c) 


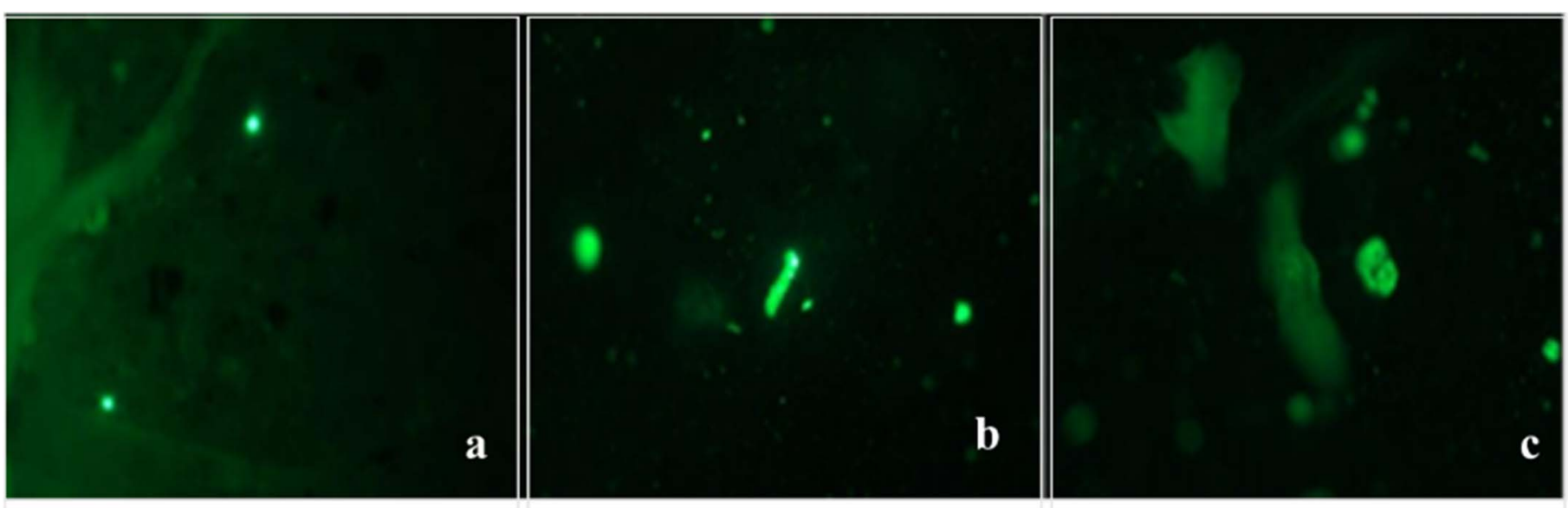

Figure 4. Effect of costaining on Oceanic soil sample with MB and NR: staining of oceanic soil sample with $200 \mu \mathrm{L}$ of NR (a), $0.6 \%$ MB and $200 \mu \mathrm{L}$ of NR (b) and $0.1 \%$ and $200 \mu \mathrm{L}$ of $\mathrm{NR}$

environmental matrices to differentiate microplastics from natural biological material. We also intend to work on designing an excitation and emission filter of the specific bandwidth to distinguish between polymer types.

\section{REFERENCES}

[1] V. Hidalgo-Ruz, L. Gutow, R. C. Thompson, and M. Thiel, "Microplastics in the marine environment: A review of the methods used for identification and quantification," Environ. Sci. Technol., vol. 46, no. 6, pp. 3060-3075, 2012, doi: 10.1021/es2031505.

[2] M. A. Browne et al., "Accumulation of microplastic on shorelines woldwide: Sources and sinks," Environ. Sci. Technol., vol. 45, no. 21, pp. 9175-9179, 2011, doi: 10.1021/es201811s.

[3] P. L. Corcoran, M. C. Biesinger, and M. Grifi, "Plastics and beaches: A degrading relationship," Mar. Pollut. Bull., vol. 58, no. 1, pp. 8084, 2009, doi: 10.1016/j.marpolbul.2008.08.022.

[4] A. L. Andrady, "Microplastics in the marine environment," Mar. Pollut. Bull., vol. 62, no. 8, pp. 1596-1605, 2011, doi: 10.1016/j.marpolbul.2011.05.030.

[5] O. S. Alimi, J. Farner Budarz, L. M. Hernandez, and N. Tufenkji, "Microplastics and Nanoplastics in Aquatic Environments: Aggregation, Deposition, and Enhanced Contaminant Transport," Environ. Sci. Technol., vol. 52, no. 4, pp. 1704-1724, 2018, doi: 10.1021/acs.est.7b05559.

[6] M. Pirsaheb, H. Hossini, and P. Makhdoumi, "Review of microplastic occurrence and toxicological effects in marine environment: Experimental evidence of inflammation," Process Saf. Environ. Prot., vol. 142, pp. 1-14, 2020, doi: 10.1016/j.psep.2020.05.050.

[7] K. J. Parton, B. J. Godley, D. Santillo, M. Tausif, L. C. M. Omeyer, and T. S. Galloway, "Investigating the presence of microplastics in demersal sharks of the North-East Atlantic," Sci. Rep., vol. 10, no. 1, pp. 1-11, 2020, doi: 10.1038/s41598-020-68680-1.

[8] Y. Chen, Y. Ling, X. Li, J. Hu, C. Cao, and D. He, "Size-dependent cellular internalization and effects of polystyrene microplastics in microalgae P. helgolandica var. tsingtaoensis and S. quadricauda," J. Hazard. Mater., vol. 399, no. June, p. 123092, 2020, doi: 10.1016/j.jhazmat.2020.123092.

[9] M. Wu, C. Yang, C. Du, and H. Liu, "Microplastics in waters and soils: Occurrence, analytical methods and ecotoxicological effects," Ecotoxicol. Environ. Saf., vol. 202, no. June, p. 110910, 2020, doi: 10.1016/j.ecoenv.2020.110910.

[10] J. P. Harrison, J. J. Ojeda, and M. E. Romero-González, "The applicability of reflectance micro-Fourier-transform infrared spectroscopy for the detection of synthetic microplastics in marine sediments," Sci. Total Environ., vol. 416, pp. 455-463, 2012, doi: 10.1016/j.scitotenv.2011.11.078.

[11] J. Rumin et al., "The use of fluorescent Nile red and BODIPY for lipid measurement in microalgae," Biotechnol. Biofuels, vol. 8, no. 1, pp. 1-16, 2015, doi: 10.1186/s13068-015-0220-4.

[12] W. Chen, C. Zhang, L. Song, M. Sommerfeld, and Q. Hu, "A high throughput Nile red method for quantitative measurement of neutral lipids in microalgae," J. Microbiol. Methods, vol. 77, no. 1, pp. 4147, 2009, doi: 10.1016/j.mimet.2009.01.001.

[13] T. Maes, R. Jessop, N. Wellner, K. Haupt, and A. G. Mayes, "A rapid-screening approach to detect and quantify microplastics based on fluorescent tagging with Nile Red," Sci. Rep., vol. 7, no. November 2016, pp. 1-10, 2017, doi: 10.1038/srep44501.

[14] W. J. Shim, Y. K. Song, S. H. Hong, and M. Jang, "Identification and quantification of microplastics using Nile Red staining," Mar. Pollut. Bull., vol. 113, no. 1-2, pp. 469-476, 2016, doi: 10.1016/j.marpolbul.2016.10.049.

[15] G. Erni-Cassola, M. I. Gibson, R. C. Thompson, and J. A. ChristieOleza, "Lost, but Found with Nile Red: A Novel Method for Detecting and Quantifying Small Microplastics (1 mm to $20 \mu \mathrm{m}$ ) in Environmental Samples," Environ. Sci. Technol., vol. 51, no. 23, pp. 13641-13648, 2017, doi: 10.1021/acs.est.7b04512.

[16] T. Stanton, M. Johnson, P. Nathanail, R. L. Gomes, T. Needham, and A. Burson, "Exploring the Efficacy of Nile Red in Microplastic Quantification: A Costaining Approach," Environ. Sci. Technol. Lett., vol. 6, no. 10, pp. 606-611, 2019, doi: 10.1021/acs.estlett.9b00499.

[17] L. Lv et al., "A simple method for detecting and quantifying microplastics utilizing fluorescent dyes - Safranine T, fluorescein isophosphate, Nile red based on thermal expansion and contraction property," Environ. Pollut., vol. 255, p. 113283, 2019, doi: 10.1016/j.envpol.2019.113283.

[18] S. Konde, J. Ornik, J. A. Prume, J. Taiber, and M. Koch, "Exploring the potential of photoluminescence spectroscopy in combination with Nile Red staining for microplastic detection," Mar. Pollut. Bull., vol. 159, no. April, p. 111475, 2020, doi: 10.1016/j.marpolbul.2020.111475.

[19] R. H. Schirmer, H. Adler, M. Pickhardt, and E. Mandelkow, "Lest we forget you - methylene blue...," Neurobiol. Aging, vol. 32, no. 12, pp. 2325.e7-2325.e16, 2011, doi: 10.1016/j.neurobiolaging.2010.12.012. S. Devalla, O. Joseph, and R. Prabhu, "Nile red-dye based analysis of synthetic fibres for forensic applications," in Proc.SPIE, Oct. 2019, vol. 11166, doi: $10.1117 / 12.2536780$. 